\title{
Thermohydrodynamic behaviors of finite journal bearings with cavitation
}

\author{
Alireza Arab Solghar ${ }^{\mathrm{a}}$ And S. A. Gandjalikhan Nassab \\ Department of Mechanical Engineering, School of Engineering, Shahid Bahonar University, Kerman, Iran
}

Received 2 October 2010, Accepted 21 October 2010

\begin{abstract}
This paper presents thermohydrodynamic solutions for a finite-width journal bearing with single axial groove on the crown by considering turbulent effects in lubricant film. Appropriate governing equations and boundary conditions are used for the fluid flow and heat transfer process occurring in finite full journal bearings. A general computer program is developed to numerically solve the set of governing equations. Results are provided for THD characteristics of journal bearing in terms of Reynolds number, clearance ratio and eccentricity ratio. Good agreement with published experimental results was achieved. The results indicate that the bearing temperature field and pressure field are considerably influenced by the journal bearing parameters.
\end{abstract}

Key words: Journal bearing / turbulent flow / simulation

\section{Introduction}

Hydrodynamic journal bearings have been widely used to support high-speed rotating machinery such as turbines, compressors and pumps. Especially, among the various types of hydrodynamic journal bearings, cylindrical journal bearings are popular because of their simple structure, relatively low manufacturing cost, and superior durability due to the high load carrying capacity and stability. Therefore, it is an important engineering problem to improve the operating characteristics of cylindrical journal bearings in the high-speed operation conditions for enhancing the quality of rotating machinery. Generally, as the operating characteristics of high-speed cylindrical journal bearings are governed by a number of bearing parameters, the bearing designers usually try to select the design variables by the conventional trial and error method using many design charts.

Milne [1] used the two-dimensional Navier-Stokes equations to derive the one dimensional lubrication equations with inertia included. Milne found that, though the two analyses differed in approach, the results of the two analyses agree in numerical detail. The one dimensional transient case for a journal bearing configuration was treated in 1970 by Fritz [2]. Extension to two-dimensional lubrication was accomplished by Smalley et al. [3], who treated both discontinuous film thicknesses and turbulence. These authors solved coupled partial differential

\footnotetext{
${ }^{a}$ Corresponding author: alireza_arabsolghar@yahoo.com
}

equations with the stream function and pressure as dependent variables.

Saibel and Macken [4] have critically reviewed the literature and Taylor and Dowson [5] have compared various turbulent lubrication theories as applicable to bearing design. Constantinescu [6], Ng and Pan [7], Ho and Vohr [8] and Hirs [9] have developed different theories for turbulence in lubricant films.

Thermal effects in turbulent lubrication were first introduced by Lund and Arwas [10]. Constantinescu [11] employed a global heat balance to derive thermal energy equations in terms of average temperatures across the film thickness. A semi-analytical solution was introduced by Safar and Szeri [12]. They assumed that the shaft is isothermal and that the bearing conducts heat in the radial direction.

A semi-analytical study of the influence of inertia terms and the effects of convection and dissipation on an infinitely long bearing was presented by Safar [13].

Hashimoto and Wada [14] developed a theoretical approach to turbulent lubrication problems including surface roughness effects based on a logarithmic velocity distribution law in the turbulent boundary layers. The three dimensional motion in the lubricant layer of a journal bearing, operating from laminar to turbulent flow conditions, was analyzed theoretically by Venkateswarlu [15]. The velocity and pressure fields were calculated from the governing differential equations using an iterative numerical method. The eddy viscosity was modeled by considering the Van Driest damping parameter. 
Nomenclature

\begin{tabular}{|c|c|}
\hline$c$ & radial clearance \\
\hline$c_{\mathrm{p}}$ & specific heat \\
\hline$D$ & bearing diameter \\
\hline$e$ & eccentricity \\
\hline$F$ & force \\
\hline$h$ & lubricant film thickness \\
\hline$h_{\text {conv }}$ & convection heat transfer coefficient \\
\hline$K$ & thermal conductivity \\
\hline$L$ & bearing width \\
\hline$n$ & normal direction \\
\hline$p$ & pressure \\
\hline $\mathrm{Pe}$ & Peclet number, $V_{\mathrm{s}} c \alpha^{-1}$ \\
\hline$Q$ & lubricant flow rate \\
\hline$r_{\mathrm{s}}$ & shaft radius \\
\hline$r_{\mathrm{i}}$ & inner radius of the bush \\
\hline$r_{\mathrm{o}}$ & bush outer radius \\
\hline$R e$ & Reynolds number, $\rho V_{\mathrm{s}} c \mu^{-1}$ \\
\hline$T$ & lubricant temperature \\
\hline$T_{\mathrm{i}}$ & inlet lubricant temperature \\
\hline$T_{\mathrm{B}}$ & bush temperature \\
\hline$T_{\mathrm{a}}$ & ambient temperature \\
\hline $\bar{u}_{i}$ & mean velocity in $i$ direction \\
\hline $\bar{u}, \bar{v}, \bar{w}$ & mean velocity components in $x$-, $y$ - and $z$-directions, respectively \\
\hline$v_{\mathrm{i}}$ & oil inlet velocity from the groove \\
\hline$V_{\mathrm{s}}$ & linear velocity of the shaft \\
\hline \multicolumn{2}{|r|}{ Greek Symbols } \\
\hline$\alpha$ & coefficient of thermal diffusivity \\
\hline$\beta$ & temperature-viscosity coefficient \\
\hline$\kappa$ & turbulence kinetic energy \\
\hline$\varepsilon$ & turbulence dissipation rate \\
\hline$\theta$ & angle in direction of rotation \\
\hline$\theta_{\mathrm{i}}$ & half angle of groove span \\
\hline$\lambda$ & ratio of groove length to bearing length \\
\hline$\mu$ & dynamic viscosity \\
\hline$\mu_{\mathrm{i}}$ & dynamic viscosity at inlet temperature \\
\hline$\nu$ & kinematic viscosity \\
\hline$\omega$ & shaft angular speed \\
\hline$\rho$ & density \\
\hline \multicolumn{2}{|r|}{ Subscripts } \\
\hline $\mathrm{a}$ & ambient \\
\hline B & bush \\
\hline cav & cavitation \\
\hline 1 & liquid \\
\hline $\operatorname{mix}$ & mixing \\
\hline rec & recirculation \\
\hline s & shaft \\
\hline side & side \\
\hline supply & supply \\
\hline$i, j$ & 1,2 and 3 denote $x$-, $y$ - and $z$-directions, respectively \\
\hline \multicolumn{2}{|r|}{ Superscripts } \\
\hline- & time average \\
\hline+ & non-dimensional form of the variables \\
\hline
\end{tabular}


A model of Reynolds stress in turbulent lubrication theory was proposed by Tieu and Kosasih [16] based on a modified Van Driest mixing length formula [17]. The theoretical analysis was used to study the effects of pressure gradient and Reynolds number on the velocity profiles.

Lee and Kim [18] brought out a physical model which combined the algebraic Reynolds stress model for the turbulent kernel and the Prandtl's mixing length theory for the near-wall regions. They predicted that the performance of finite journal bearing operated in turbulent regime can change greatly depending on both the turbulence model and the cavitation boundary condition.

In 2004, Chun and $\mathrm{Ha}$ [19] considered the effects of variable density and variable specific heat on maximum pressure and maximum temperature in high-speed journal bearing operation. They used the Reynolds equation for computing lubricant pressure in a steadily loaded journal bearing with infinite width under turbulent condition. Through the result of analysis, they concluded that under high speed operations, the consideration of variable density and variable specific heat on the calculation of bearing load and frictional power loss cannot be ignored.

Peng and Khonsari in 2006 [20] developed a THD model for predicting the three-dimensional temperature field in an air-lubricated journal bearing. In that study, they used simultaneous solution of Reynolds equation and the energy equation for air flow in the gas bearing. Parametric studies covering a fairly wide range of operating speeds and load conditions were carried.

However, from this review it is clear that, as far as the authors are aware, in the most of previous theoretical works the classical theory of lubrication was considered that inertia forces should be negligible against viscous stresses and pressure forces; however in journal bearings, the inertia forces might become preponderant due to small kinematic viscosity and due to high linear speed of rotating shaft. The presence of inertia forces in lubricant films does not allow rigorous use of the Reynolds equation for bearing calculations. On the other hand, when the inertia forces reach a certain value, they change the flow structure that becomes, progressively, turbulent. Consequently, when the Reynolds number, is increasing, the Taylor's vortex pattern flow and the turbulent regime may occur. Thus, the main goal of the present work is to provide a comprehensive set of data for the steady state three-dimensional THD characteristics of journal bearings running under turbulent conditions. So the full Navier-Stokes equations, turbulent kinetic energy and its dissipation rate equations are solved along with the energy equation in the lubricant flow and the heat conduction equation of bush and shaft to obtain the steady state characteristics. The turbulent flow inside the journal bearing is modelled using a modified low-Re $k-\varepsilon$ turbulence model [21]. The model can reproduce the near-wall limiting behavior and provides accurate predictions for the boundary layer of turbulent flows with favorable or adverse pressure gradients such as flow of the lubricant in journal bearings. The problem is formulated mathematically and solved numerically using the computational fluid dynamics (CFD) approach with appropriate boundary conditions. A suitable cavitation model is applied for considering cavitation effects. In order to investigate the influence of main parameters on the steady state performance characteristics of journal bearings for a variety of simulated operating conditions, different values of eccentricity, radial clearance and shaft rotation speed are considered. The computational results are in good agreement with the experimental and theoretical findings.

\section{Formulation}

For a three-dimensional, steady state, turbulent flow with incompressible Newtonian fluid, the dimensionless form of the continuity and Navier-Stokes equation may be written as follows:

$$
\begin{gathered}
\left(\bar{U}_{i}^{+}\right)_{, i}=0 \\
\left(\bar{U}_{i}^{+} \bar{U}_{j}^{+}\right)_{, j}=-\bar{p}_{, i}^{+}+\frac{1}{R e}\left[\left(\mu^{+}\right)\left(\bar{U}_{i, j}^{+}+\bar{U}_{j, i}^{+}\right)-\overline{u_{i} u_{j}}\right]_{, j}
\end{gathered}
$$

The term $\overline{u_{i} u_{j}}$ appears on the right hand side of Equation (2) is the Reynolds stress tensor. By using Boussinesq assumptions which links the Reynolds stresses to the velocity gradients via the turbulent dynamic viscosity:

$$
\rho \overline{u_{i} u_{j}}=-\mu_{t}\left(\bar{U}_{i, j}+\bar{U}_{j, i}\right)+\frac{2}{3} \delta_{i j} \rho k
$$

and inserting Equation (2) in Equation (3), after some mathematical manipulations, yields:

$$
\begin{aligned}
\left(\bar{U}_{i}^{+} \bar{U}_{j}^{+}\right)_{, j}=-\left(\bar{p}^{+}\right. & \left.+2 k^{+}\right)_{, i}+\frac{1}{R e} \\
& \times\left[\left(\mu^{+}+\mu_{t}^{+}\right)\left(\bar{U}_{i, j}^{+}+\bar{U}_{j, i}^{+}\right)\right]_{, j}
\end{aligned}
$$

In the above equation, $i, j$ equals 1,2 and 3 denote $x$-, $y$ and $z$-directions, respectively.

In order to study the thermal behaviors of journal bearings, the energy equation and heat conduction equations should be solved in the lubricant film and bearing, respectively. The nondimensional form of the energy equations in the flow field and solid element may be written as shown below:

$$
\begin{gathered}
\left(\bar{U}_{j}^{+} \bar{T}^{+}\right)_{, j}=\left[\left(\frac{1}{P e}+\frac{\mu_{t}^{+}}{\delta_{T} R e}\right) \bar{T}_{, j}^{+}\right]_{, j}+\frac{\mu^{+} c}{r_{\mathrm{s}}} \\
\times\left(\bar{U}_{i, j}^{+}+\bar{U}_{j, i}^{+}\right) \bar{U}_{i, j}^{+} \\
\left(T_{\mathrm{B}, j}^{+}\right)_{, j}=0
\end{gathered}
$$

Owing to the high viscous dissipation rate, the dynamic viscosity must be taken into account as a function of temperature, according to the following common form

$$
\mu=\mu_{\mathrm{i}} \mathrm{e}^{-\beta\left(T-T_{\mathrm{i}}\right)}
$$


In this expression $\beta=0.032$ is the temperature-viscosity coefficient of the lubricant and $T_{\mathrm{i}}$ is the inlet lubricant temperature.

A turbulence model is required to determine the turbulent shear stress existing in the governing equations. The present analysis uses the AKN low Re $\kappa-\varepsilon$ turbulence model (Abe et al. [21]). This model falls into the class of eddy viscosity models. Two transport equations are derived which describe transport of the turbulent kinetic energy and its dissipation. The Reynolds stress tensor is then computed using an assumption which relates the Reynolds stress tensor to the velocity gradients and an eddy viscosity. The latter is obtained from the two transported scalars. The following equations are used as transport equation for $k-\varepsilon$ :

$$
\begin{aligned}
&\left(\bar{U}_{j}^{+} k^{+}\right)_{, j}= {\left[\frac{1}{R e}\left(1+\frac{\mu_{t}^{+}}{\sigma_{k}}\right) k_{, j}^{+}\right]_{, j}+\frac{\mu_{t}^{+}}{R e} } \\
& \times\left(\bar{U}_{i, j}^{+}+\bar{U}_{j, i}^{+}\right) \bar{U}_{i, j}^{+}-\varepsilon^{+} \\
&\left(\bar{U}_{j}^{+} \tilde{\varepsilon}^{+}\right)_{, j}=\left[\frac{1}{R e}\left(1+\frac{\mu_{t}^{+}}{\sigma_{\varepsilon}}\right) \tilde{\varepsilon}_{, j}^{+}\right]_{, j}+\frac{C_{\varepsilon 1} \mu_{t}^{+}}{R e} \\
& \times\left(\bar{U}_{i, j}^{+}+\bar{U}_{j, i}^{+}\right) \bar{U}_{i, j}^{+}-C_{\varepsilon 2} f_{\varepsilon} \frac{\tilde{\varepsilon}^{+2}}{k^{+}}
\end{aligned}
$$

in which

$$
\varepsilon^{+}=\tilde{\varepsilon}^{+}+\frac{2}{R e}\left(\frac{\partial \sqrt{k^{+}}}{\partial n}\right)^{2}
$$

where $n$ is the direction normal to the journal surface. The non-dimensional forms of the damping functions $f_{\mu}$ and $f_{\varepsilon}$ are given as follows:

$$
\begin{aligned}
& f_{\mu}=\{[1-\left.\left.\exp \left(-\frac{R e^{0.75} \varepsilon^{+0.25} y_{s}}{14}\right)\right]\right\}^{2} \\
& \times {\left[1+\frac{5}{R_{t}^{3 / 4}} \exp \left\{-\left(\frac{R e k^{+2}}{200 \varepsilon^{+}}\right)^{2}\right\}\right] } \\
& f_{\varepsilon}=\left\{\left[1-\exp \left(-\frac{R e^{0.75} \varepsilon^{+0.25} y_{s}}{3.1}\right)\right]\right\}^{2} \\
& \times\left[1-0.3 \exp \left\{-\left(\frac{R e k^{+2}}{6.5 \varepsilon^{+}}\right)^{2}\right\}\right]
\end{aligned}
$$

and $y_{\mathrm{s}}$ is the normal distance of each nodal point to the nearest solid boundary that damps the turbulence kinetic energy. In addition, the dimensionless form of turbulent dynamic viscosity may be written as

$$
\mu_{t}^{+}=R e C_{\mu} f_{\mu} \frac{k^{+2}}{\varepsilon^{+}}
$$

According to the present turbulence model, the standard model constants $\sigma_{k}, \sigma_{\varepsilon}, \sigma_{T}, C_{\mu}, C_{\varepsilon 1}$ and $C_{\varepsilon 2}$ are 1.4,
Table 1. Nondimensional variables.

\begin{tabular}{lc}
\hline Dimensional parameters & Normalizing parameter \\
\hline Linear coordinates $x i$ & $c$ \\
Film velocities components $U i$ & $V_{\mathrm{s}}$ \\
Turbulent kinetic energy $k$ & $V_{\mathrm{s}}^{2}$ \\
Turbulent dissipation rate $\varepsilon$ & $V_{\mathrm{s}}^{3} / c$ \\
Pressure $p$ & $\rho V_{\mathrm{s}}^{2}$ \\
Dynamic viscosity $\mu$ & $\mu_{i}$ \\
Turbulent dynamic viscosity $\mu_{t}$ & $\mu_{i}$ \\
Kinematic viscosity & $\nu$ \\
Temperature $T$ & $\mu_{i} \omega\left(R_{\mathrm{s}} / c\right)^{2} / \rho c_{\mathrm{p}}$ \\
\hline
\end{tabular}

1.4, 0.9, 0.09, 1.4 and 1.9, respectively. Moreover, the dimensional and normalizing quantities are designated in Table 1 .

Also the Reynolds and Peclet numbers are defined as $R e=\rho V_{\mathrm{s}} c / \mu$ and $P e=V_{\mathrm{s}} c / \alpha$, respectively. In order to solve the coupled differential equations of the problem, appropriate boundary conditions should be implemented, thus the boundary conditions for the above differential equations are summarized in Figure 1.

It is noteworthy that in journal bearing the cyclic variation of the shaft temperature is small and the shaft may be considered as an isothermal surface whose temperature is much closer to the mean lubricant temperature on bearing inner surface [22]. Hence, at each axial section the shaft temperature is computed as follow:

$$
\left.T_{\mathrm{s}}=\frac{1}{2 \pi} \int_{0}^{2 \pi} \bar{T}\right)_{r=r_{\mathrm{i}}} \mathrm{d} \theta
$$

where $\bar{T})_{r=r_{\mathrm{i}}}$ denotes the variation of circumferential lubricant temperature on the bearing inner surface at each axial section.

In the vicinity of inlet groove, the temperature of recirculating fluid, $T_{\text {rec }}$, is naturally higher than the temperature of the incoming supply lubricant, $T_{\text {supply }}$. Thus, the recirculating flow transfers a portion of its energy to the supply oil at the inlet. An energy balance at the inlet gives (Heshmat and Pinkus [23], Khonsari et al. [24]):

$$
\bar{T}_{\text {mix }}=\frac{\bar{T}_{\text {rec }} Q_{\text {rec }}+\bar{T}_{\text {supply }} Q_{\text {supply }}}{Q_{\text {rec }}+Q_{\text {supply }}}
$$

where $Q_{\text {rec }}$ and $Q_{\text {supply }}$ are given by the following equations

$$
\begin{aligned}
Q_{\text {rec }} & =\int_{\Delta z} \int_{l\left(\pi-\theta_{i}\right)}^{r_{i}} \bar{V}_{\theta}\left(r, \pi-\theta_{i}, z\right) \mathrm{d} r \mathrm{~d} z \\
Q_{\text {supply }} & =\int_{\Delta z}^{2 \theta_{i}} v_{i} r_{i} \mathrm{~d} z
\end{aligned}
$$

In addition, at the groove location, the source term in the energy equation is disregarded across the lubricant film. 


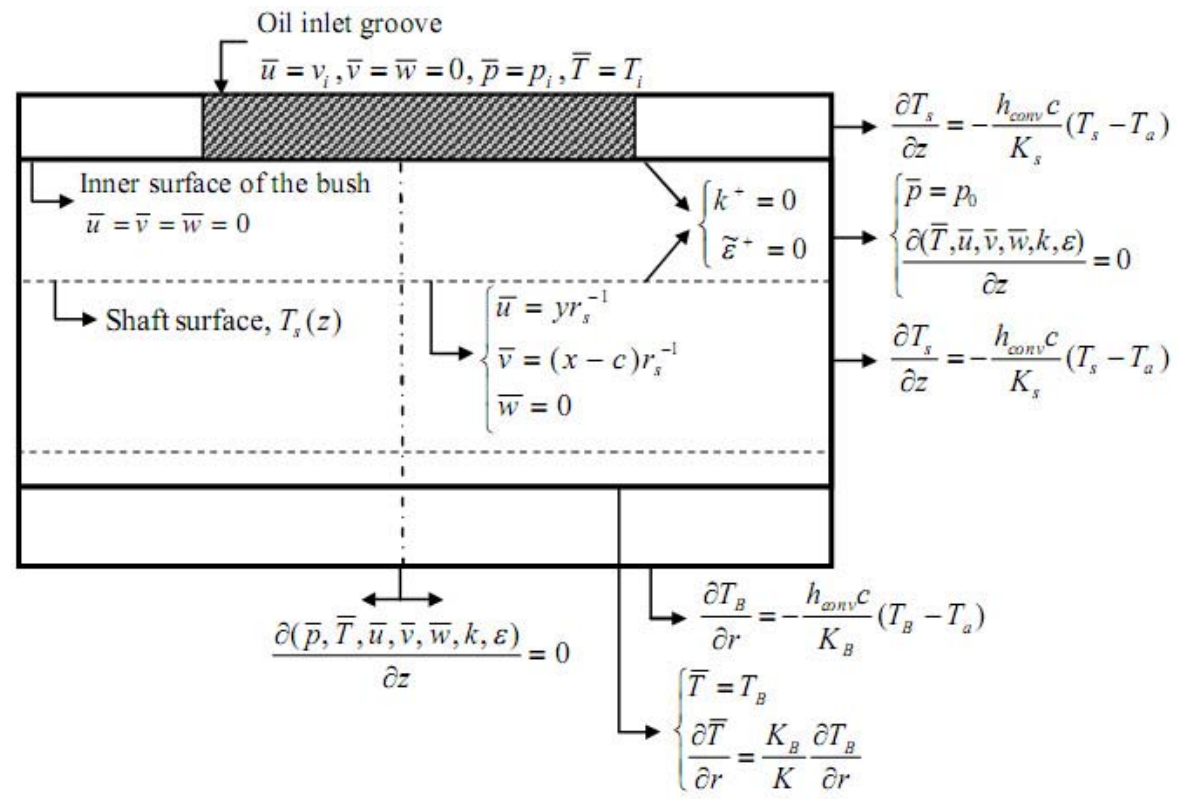

Fig. 1. The boundary conditions of the problem.

Furthermore, for all dependent variables, periodic boundary condition in circumferential direction is implemented as follows:

$$
(\bar{p}, \bar{T}, \bar{U}, \bar{V}, \bar{W}, k, \varepsilon)_{\theta=0}=(\bar{p}, \bar{T}, \bar{U}, \bar{V}, \bar{W}, k, \varepsilon)_{\theta=2 \pi}
$$

$$
\begin{gathered}
\left(\frac{\partial(\bar{p}, \bar{T}, \bar{U}, \bar{V}, \bar{W}, k, \varepsilon)}{\partial \theta}\right)_{\theta=0}= \\
\quad\left(\frac{\partial(\bar{p}, \bar{T}, \bar{U}, \bar{V}, \bar{W}, k, \varepsilon)}{\partial \theta}\right)_{\theta=2 \pi}
\end{gathered}
$$

Besides, in order to calculate the inlet oil velocity $v_{\mathrm{i}}$ at each iteration, firstly, the lubricant side leakage is computed as

$$
Q_{\text {side }}=\int_{r_{\mathrm{B}}}^{r_{\mathrm{B}}} \int_{0}^{2 \pi} \bar{W} \mathrm{~d} r \mathrm{~d} \theta
$$

in which, $\bar{W}$ is the axial velocity component at the outlet section, and secondly the oil entering flow rate from the inlet hole must be equal to the lubricant flow rate leaving the bearing, such that at each iteration, the inlet oil velocity is modified as:

$$
v_{i}=\frac{Q_{\text {side }}}{r_{i} \theta_{i} \lambda L}
$$

In the above equation $\lambda$ is slenderness ratio, i.e., the ratio of groove width to bearing width.

The following is the two equations for the components of the pressure force along the line of center and normal to it.

$$
F_{x}=\int_{-L / 2}^{L / 2} \int_{0}^{R \theta_{\text {cav }}} p \cos \theta \mathrm{d} \theta \mathrm{d} z
$$

$$
F_{y}=\int_{-L / 2}^{L / 2} \int_{0}^{R \theta_{\text {cav }}} p \sin \theta \mathrm{d} \theta \mathrm{d} z
$$

Therefore, the attitude angle, i.e., the angle between the load vector and the line of center is determined from the two load components as:

$$
\psi=\tan ^{-1}\left|\frac{F_{y}}{F_{x}}\right|
$$

\section{Cavitation modelling}

During iterative solution, whenever the pressure falls below the value of cavitation pressure at a grid point, a cavitation algorithm is considered. By this method, the approximate boundaries locations of the cavitated region at any axial section are determined at each iteration level. In the cavitated region based on the experimental observation by Heshmat [25], there are two different parts; narrow oil streamlets extending over the gap and between the film rupture and reformation boundary, and a layer of lubricant adhering to the journal and moving uniformly with the journal speed (Fig. 2). Such detached pattern suggests that there is a very weak bond between the moving streamlets and the stationary surface, permitting side entrainment of gas to take place in the cavitated region.

Since, the range of cavitated domain can be distinguished with the values of lubricant pressure, it is necessary to solve the governing equations for obtaining the lubricant pressure in the whole domain. Therefore, in the applied cavitation model, to solve the governing equations that are written for a single phase fluid, an attempt is made to substitute an equivalent fluid in the cavitated 


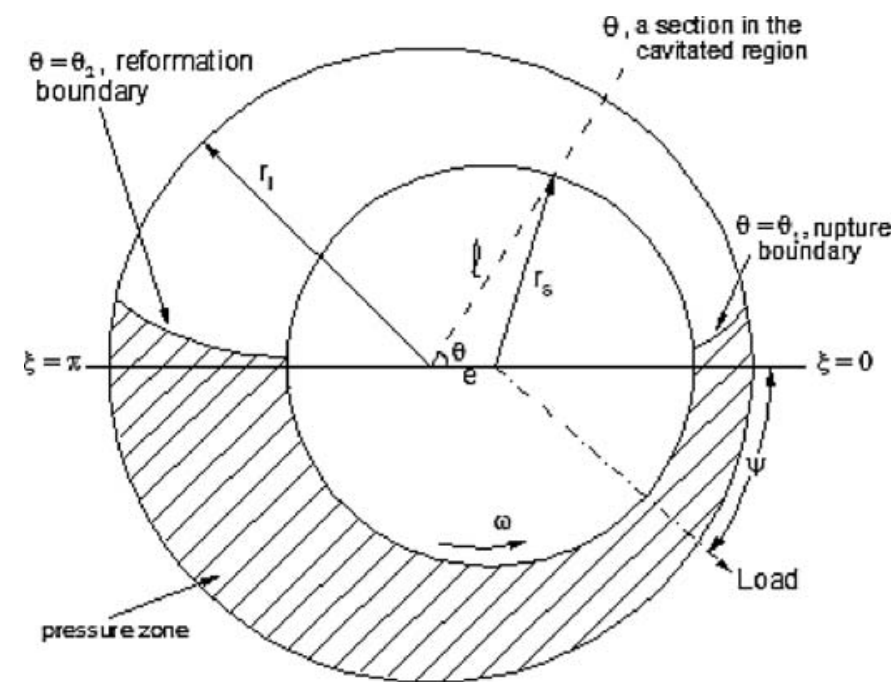

Fig. 2. The film rupture and reformation boundaries of the cavitated region at each axial section.

region. To formulate this model, it is assumed that a homogenous mixture of lubricant and vapor exists in the cavitated zone with mean physical properties depending on the fraction of liquid and vapor. The liquid fraction $\gamma$, is computed on the basis of continuity requirements. This parameter is defined as the volume of liquid to lubricant volume such that one can compute the density of mixture in the cavitated region by:

$$
\rho=\gamma \rho_{\ell}+(1-\gamma) \rho_{v}
$$

The value of $\gamma$ varies in circumferential direction, such that $\gamma=1$ for the uncavitated part. Now, other mean properties of the mixture such as dynamic viscosity and thermal conductivity can be calculated as:

$$
m=\frac{m_{\ell} m_{\nu}}{m_{\ell}-\gamma\left(m_{\ell}-m_{\nu}\right)}
$$

For computing the value of $\gamma$ based on continuity requirements, a two-dimensional continuity equation is applied. For the purpose of illustration and referring to Figures 2 and 3 , if $h(\theta)$ and $h_{\ell}(\theta)$ are the total film thickness and the liquid film thickness, respectively, then the lubricant mass flow rate with the assumption of uniform velocity distribution for the liquid layer and linear one for the vapor flow is computed as follows:

$$
\dot{m}_{\theta}=\rho_{\ell} \bar{V}_{\mathrm{s}} h_{\ell}(\theta)+\rho_{v} \frac{\bar{V}_{\mathrm{s}}}{2}\left[h(\theta)-h_{\ell}(\theta)\right]
$$

which leads to the following equation for computing the liquid film thickness

$$
h_{\ell}(\theta)=\frac{\dot{m}_{\theta}-0.5 \bar{V}_{\mathrm{s}} \rho_{v} h(\theta)}{\bar{V}_{\mathrm{s}} \rho_{\ell}-0.5 \bar{V}_{\mathrm{s}} \rho_{v}}
$$

where $\dot{m}_{\theta}$ is a known parameter which can be computed in the uncavitated region by

$$
m=\int_{\ell\left(\theta_{1}-\Delta \theta\right)}^{r_{i}} \rho_{\ell} V_{\theta}\left(\theta_{1}-\Delta \theta, r\right) \mathrm{d} r
$$
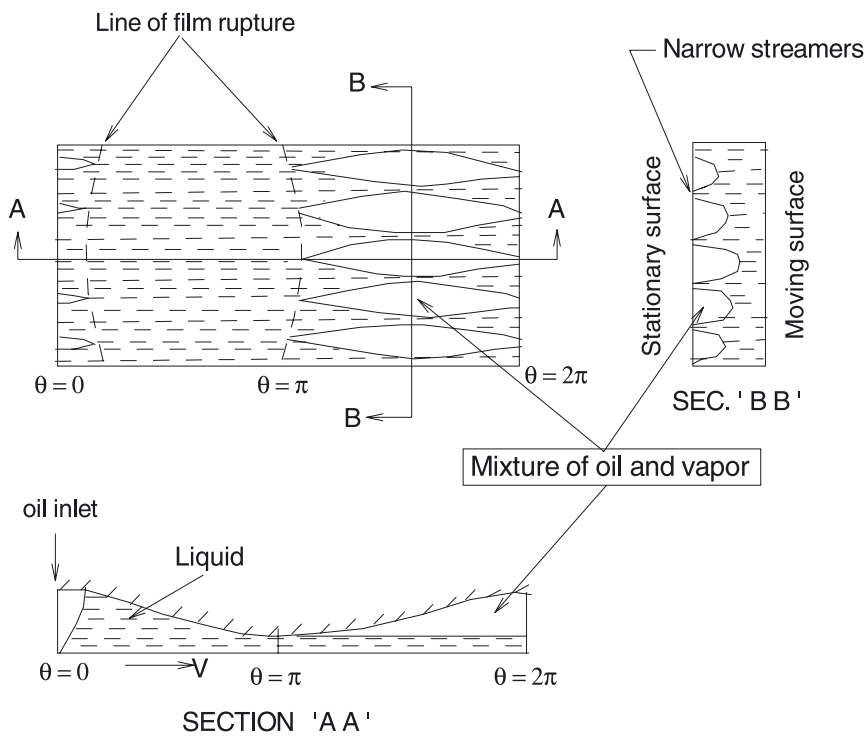

Fig. 3. The schematic of cavitation.

After computing the value of the liquid film thickness from Equation (28), the liquid fraction in the cavitated part of the lubricant flow can be obtained by:

$$
\gamma=\frac{h_{\ell}(\theta)}{h_{\ell}(\theta)+0.5\left[h(\theta)-h_{\ell}(\theta)\right]}
$$

Consequently, using mean properties of the mixture in the cavitated region, the complete set of governing equations those are the continuity, momentum, energy, turbulent kinetic energy and dissipation rate are solved in the entire flow domain at each iteration. As the numerical solution of momentum equation resulted in a negative absolute pressure in the cavitated region, the pressure is set equal to zero absolute pressure as the value of cavitation pressure for gaseous cavitation.

\section{The procedure of computation}

The nondimensionalized governing equations are discretized by using hybrid scheme and numerically solved using the finite volume method and the SIMPLE algorithm. A computer program written in a modular fashion in FORTRAN was developed.

Numerical solutions are obtained iteratively by the line-by-line method progressing in axial direction. As the result of grid tests for obtaining the grid-independent solutions, an optimum grid of $150 \times 60 \times 30$ in circumferential, radial and axial direction, respectively, with clustering near the journal surface, was used for the flow field calculations and a uniform grid of the same size was used for the bearing. Also, the same grid size with clustering near the journal surface was used for the shaft. The iterations were terminated when the sum of the absolute residuals was less than $10^{-4}$ for each equation.

The iterations are repeated until the entire system converged. Figure 4 depicts the flow chart of the computer program. 


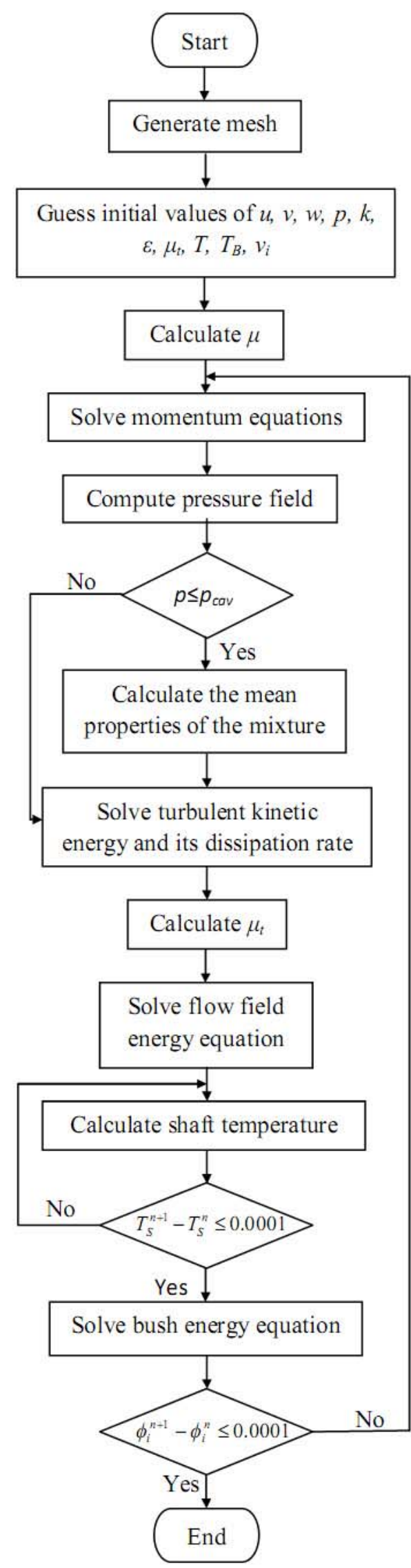

Fig. 4. Flow chart of computer program.

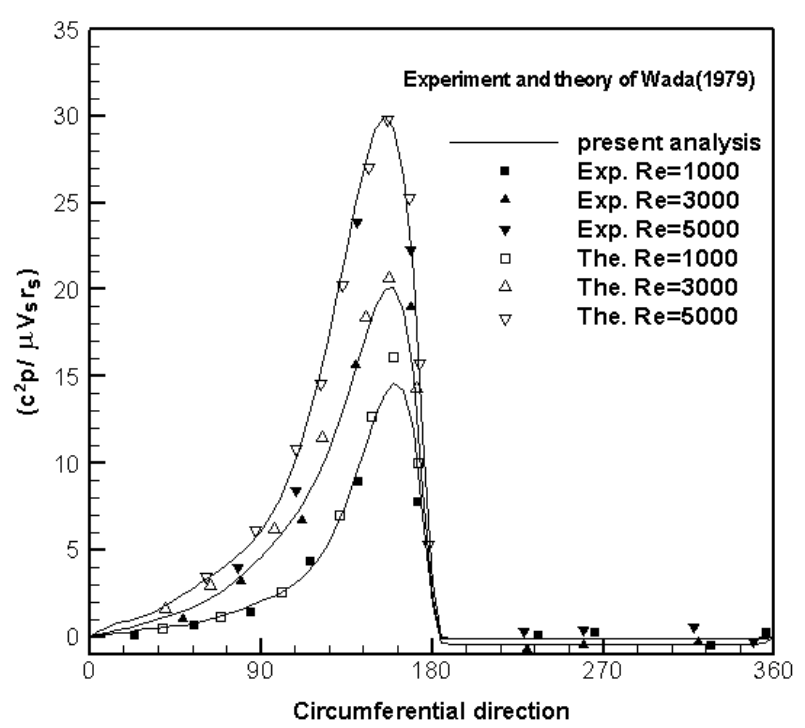

Fig. 5. The comparison between pressure distribution of present work with theoretical and experimental $\operatorname{data}(e / c=$ $\left.0.84, c / r_{\mathrm{s}}=0.0088\right)$.

\section{Results}

In the present work, the set of governing equations for turbulent lubricant flow in journal bearings were numerically solved to obtain the THD characteristics of such bearings. In the first step, to examine the validity of the numerical results, computations were carried out for a test case and the computed results were compared with experimental data and with the theoretical results of Wada and Hashimoto [26]. Figure 5 shows the pressure distribution in the circumferential direction on the shaft surface. Obviously, the generation of hydrodynamic pressure in the converging zone is seen in this figure such that the maximum pressure takes place in a section upstream to the minimum film thickness. However, Figure 5 shows that there is a satisfactory consistency between the computed pressure distribution with experiment and also with theory. Moreover, the following results are THD characteristics of journal bearings under turbulent flow running under severe operating conditions. The aforementioned bearings have axial grooves located on the line of centers at the section of maximum gap.

In the journal bearing, the Reynolds number, clearance ratio and eccentricity ratio are the main three parameters such that the THD characteristics of such bearing are markedly affected by these parameters. In order to study the effects of these parameters on the hydrodynamic and thermal behaviors of journal bearings, the variation of bush inner surface temperature and also the distribution of the lubricant pressure on the shaft surface at the mid-plane of the bearing are plotted in Figures 6 and 7 for different values of Reynolds number, clearance ratio and eccentricity ratio such that Figures $6 \mathrm{a}$, b and $\mathrm{c}$ display the pressure distribution, and that Figures $7 \mathrm{a}, \mathrm{b}$ and $\mathrm{c}$ display temperature distributions. 


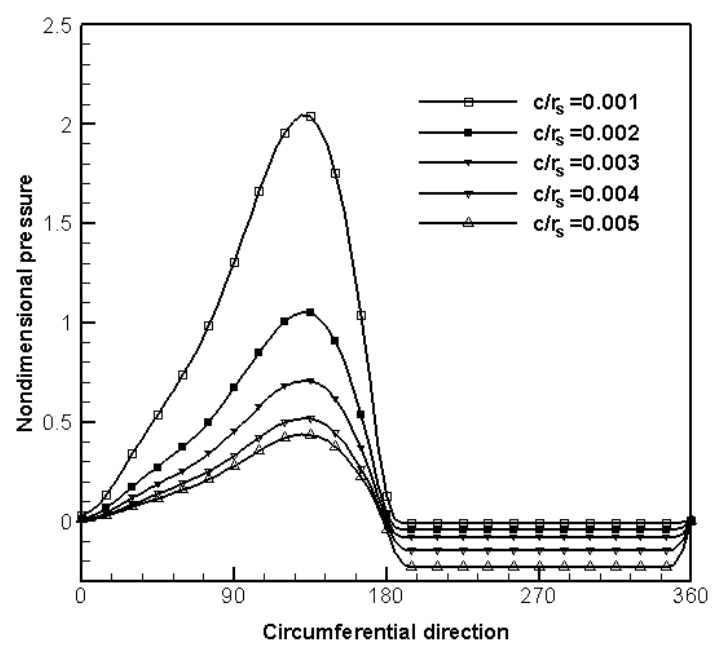

(a)

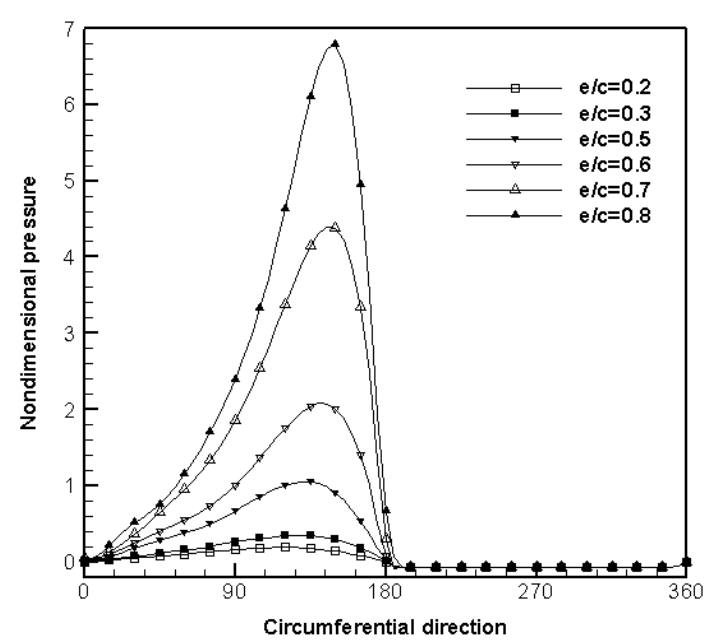

(b)

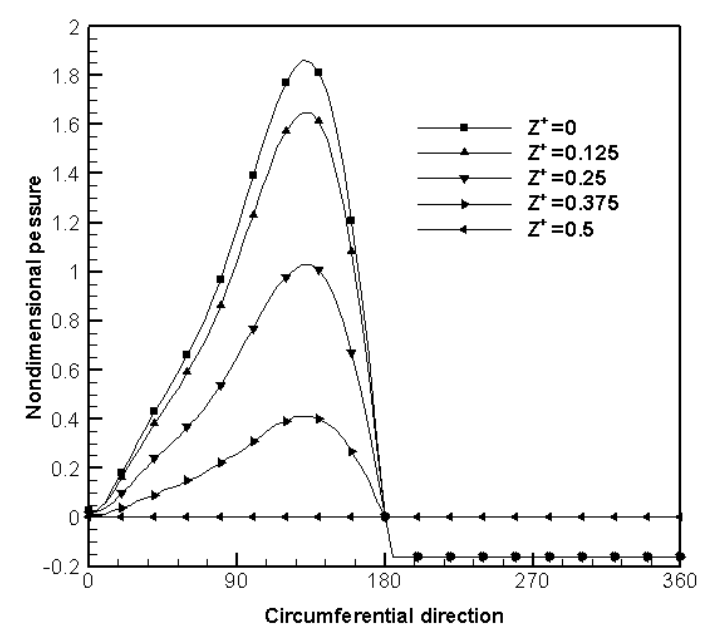

(c)

Fig. 6. (a) Circumferential pressure distribution for various clearance ratios $(R e=8000, e / c=0.4)$. (b) Effect of eccentricity ratio on the lubricant pressure $\left(R e=8000, c / r_{\mathrm{s}}=0.003\right)$. (c) Influence of Reynolds number on the axial lubricant pressure $(e / c=0.4)$.
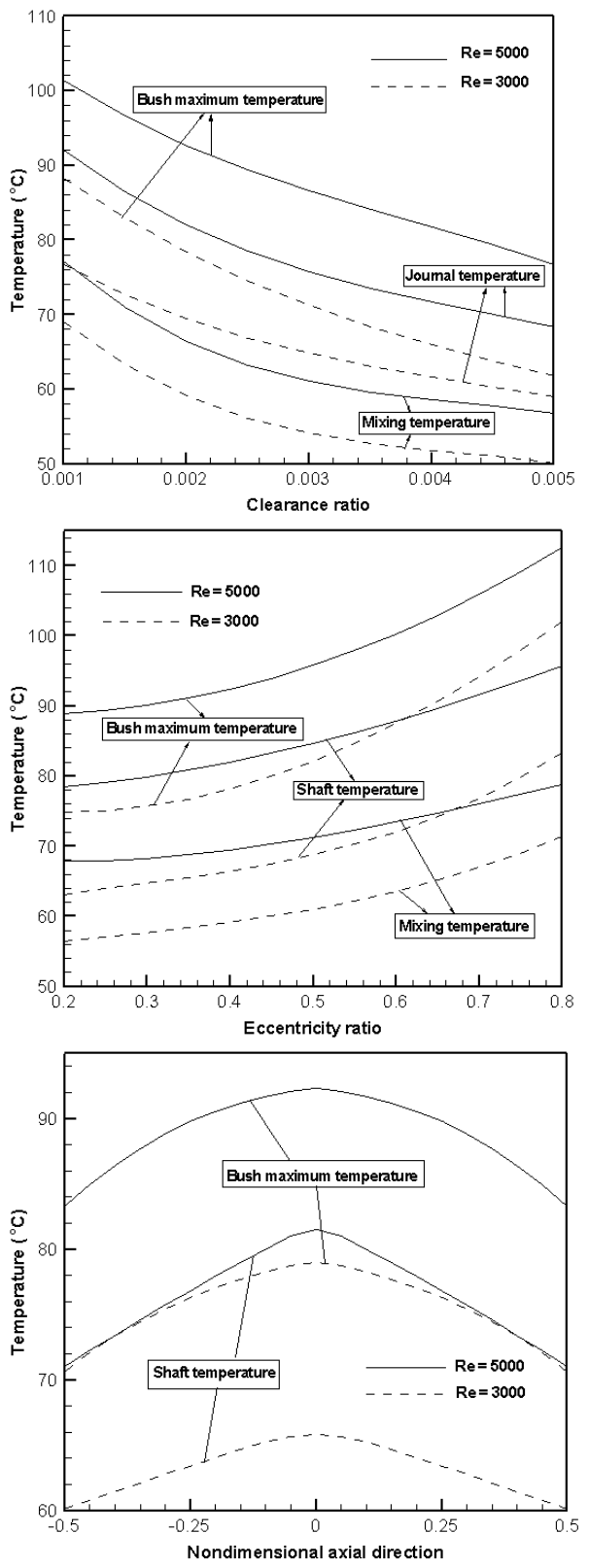

Fig. 7. (a) Maximum bush, shaft and mixing temperature variation versus clearance ratio $(e / c=0.4)$. (b) Maximum bush, shaft and mixing temperature variation versus eccentricity ratio $\left(c / r_{\mathrm{s}}=0.002\right)$. (c) Shaft and maximum bush temperature along axial direction $\left(e / c=0.4, c / r_{\mathrm{s}}=0.002\right)$.

The generation of hydrodynamic pressure in the journal bearing is clearly observed such that Figure 3 reveals a rapid increase in the lubricant pressure as the fluid passes through the converging zone of the bearing. The lubricant maximum pressure occurs near the minimum gap after which the lubricant pressure decreases sharply. The extend of cavitated domain can be easily distinguished in which the magnitude of lubricant pressure is equal to the vapor pressure.

To investigate the effects of clearance ratio and bearing eccentricity ratio on the hydrodynamic behavior of 
journal bearing, the distributions of lubricant pressure on the shaft surface at the mid-plane are plotted in Figures $6 \mathrm{a}$ and $\mathrm{b}$ for different values of the parameters $e / c$ and $c / r_{\mathrm{s}}$. These figures display that the value of hydrodynamic pressure generated in the pressure zone increases with increasing in $e / c$ and decreasing in $c / r_{\mathrm{s}}$, such that, the larger eccentricity and smaller clearance ratio, the higher load capacity. In addition, the effect of bearing eccentricity on the angular position of maximum lubricant pressure is that this point varies towards the minimum film thickness in the direction of shaft rotation.

The variation of lubricant maximum pressure for different values of axial section are presented in Figure 6c. This figure illustrates that the value of hydrodynamic pressure decreases along the bearing from the mid-plane towards the end section. Besides, the phenomena of pressure increase with decreasing in $e / c, c / r_{\mathrm{s}}$ and Reynolds number is apparently seen in Figures 5 and 6 . However, in comparing the various pressures curves, one should recall that the pressure is normalized by the factor $\rho V_{\mathrm{s}}^{2}$, which differs numerically from one curve to the next. Consequently, the lubricant pressure $p=\rho V_{\mathrm{s}}^{2} p^{+}$increases with increasing the Reynolds number.

The influence of clearance ratio on the temperature fields in the journal bearings is shown in Figure 7a. This figure demonstrates the profound effect of clearance ratio on the variation of temperature in the journal bearings. Apparently, the shaft temperature is constantly lower than the maximum temperature of the bush and higher than the minimum temperature of the bush and mixing temperature.

The temperature field of the journal bearing is significantly affected by eccentricity ratio as shown in Figure 7b, such that whenever the shaft runs more eccentric results higher temperature of the surface of journal and bush.

In order to enhance our understanding of the thermal behavior of the journal bearings, the variation of lubricant maximum temperature and the journal temperature for different values of the Reynolds number along the axial direction are depicted in Figure 7c. This figure reveals that the value of maximum temperature of the bush and also the temperature of the shaft decrease along the bearing from the mid-plane towards the two end sections due to the cooling effect of inlet oil.

One of the main parameters which has a significant effect on THD characteristics of journal bearing is the lubricant side leakage. This lubricant axial flow rate has the key role in the cooling and heat dissipation removing from the bearing system.

The effect of eccentricity ratio and clearance ratio on the lubricant side leakage for two different values of $R e$ is shown in Figures $8 \mathrm{a}$ and b, respectively. These figures exhibit that the lubricant flow rate increases by increasing in $e / c, c / r_{\mathrm{s}}$ and Reynolds number. One should recall that in the computations of Figures 6, the lubricant side leakage is non-dimensionalized by the parameter $V_{\mathrm{s}} c L$.

The variation of the locus of the journal center as a function of clearance ratio and Reynolds number is shown in Figures 9a and b. It can be observed that when
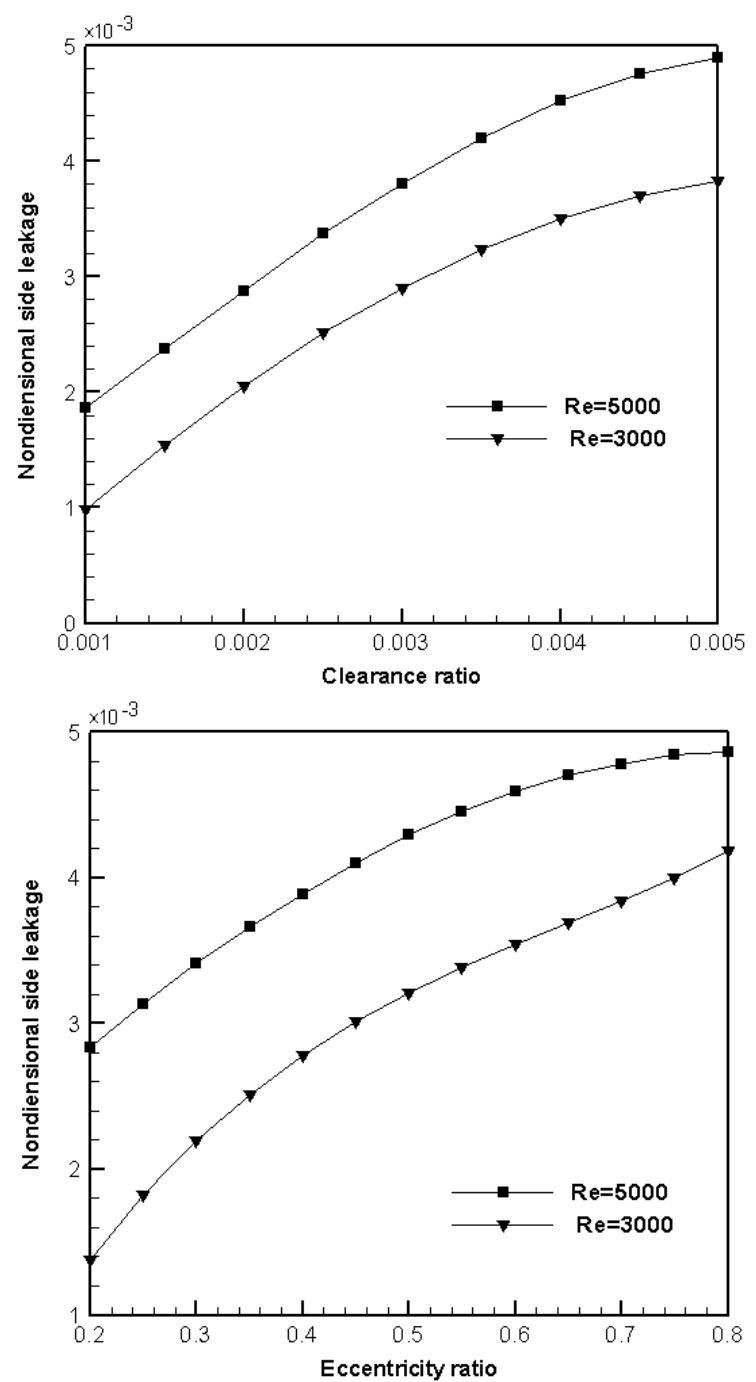

Fig. 8. (a) Side leakage as a function of clearance ratio $(e / c=$ $0.6)$. (b) Side leakage as a function of eccentricity ratio $\left(c / r_{\mathrm{s}}=\right.$ $0.004)$.

eccentricity ratio increases attitude angle decreases, this means that load carrying capacity of bearing has improved. In addition, the loci of the journal surface is not significantly affected by the variation of Reynolds number and clearance ratio, however the attitude angle increases with rising in value of those parameters.

\section{Discussion}

The present paper dealt with the numerical simulation of the flow and heat transfer inside the journal bearing. Present computational approach is valuable to the practical modeling of the journal bearing operating under turbulent regime and in preparing the design charts in prediction of both hydrodynamic and thermal behaviors of journal bearings. The three dimensional THD characteristics of journal bearings with single axial groove located on maximum film thickness, operating under turbulent 

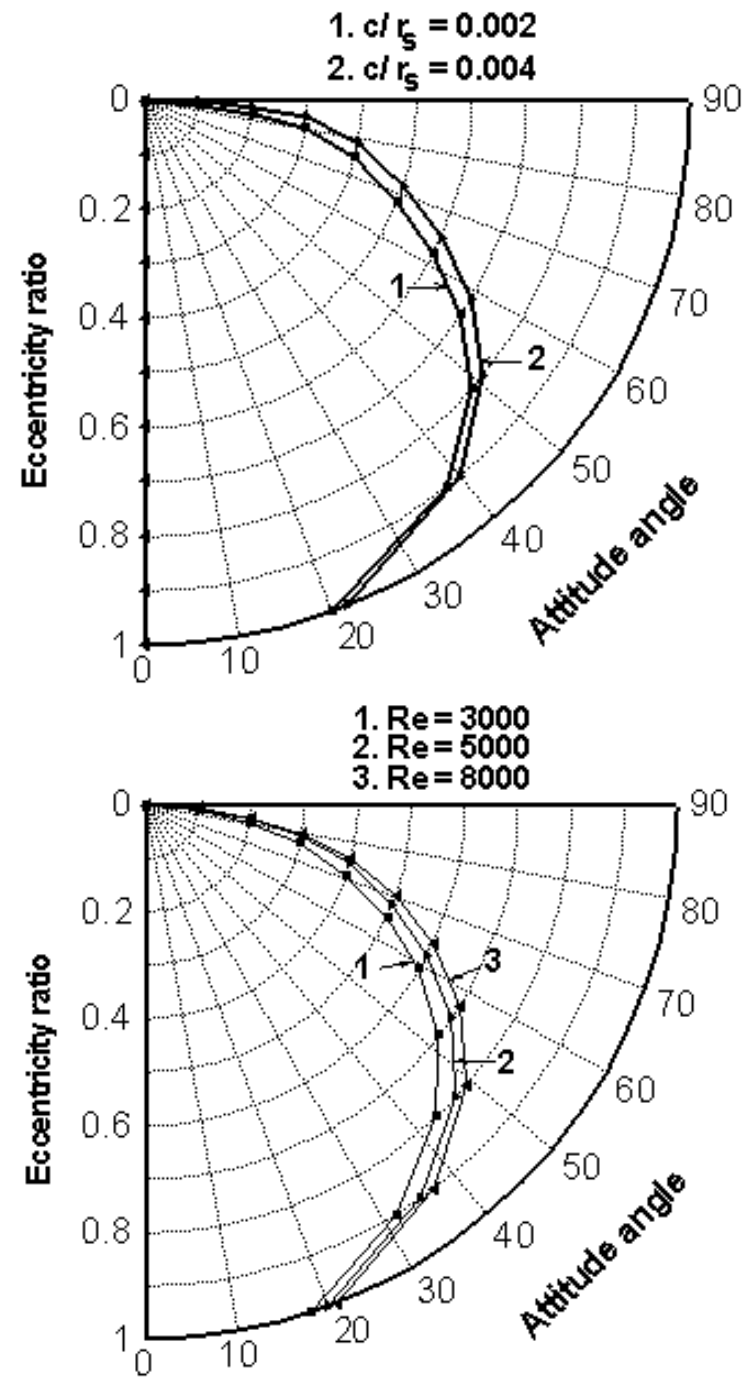

Fig. 9. (a) Locus of journal center as a function of clearance ratio. (b) Locus of journal center as a function of Reynolds number $\left(c / r_{\mathrm{s}}=0.002\right)$.

regime was modeled by using $\mathrm{CFD}$ approach. Besides, cavitation phenomenon was considered in the computation. Comparing the simulated and experimental data indicated an acceptable agreement, the following conclusions can be drawn:

(1) The predicted performance of finite journal bearing operated in turbulent condition can considerably change depending on the Reynolds number, eccentricity and clearance ratio.

(2) The magnitude of maximum oil pressure on the journal surface is considerably alternated by eccentricity and clearance ratio.

(3) The bush and shaft temperature varies in which by axial direction, which indicates the cooling effect in the journal bearings.

(4) The amount of side leakage is dramatically affected by $R e, e / c$, and $c / r_{s}$, in which it increases when
Reynolds number, eccentricity ratio and clearance ratio increase.

(5) The loci of the shaft center varies as journal parameters vary.

\section{References}

[1] A.A. Milne, On the effect of lubricant inertia in the theory of hydrodynamic lubrication, ASME J. Basic Eng. 81 (1959) 239

[2] R.J. Fritz, The effect of an annular fluid on the vibration of a long rotor; Part I, Theory, ASME J. Basic Eng. (1970) 923-929

[3] A.J. Smalley, J.H. Vohr, V. Castelli, C. Wachmann, An analytical and experimental investigation of turbulent flow in bearing films involving convective fluid inertia forces, ASME Paper 73-LubS-l (1973)

[4] E.S. Saibel, N.A. Macken, Nonlaminar behavior in bearings: a critical review of the literature, J. Lubr. Technol. 96 (1974) $174-181$

[5] C.M. Taylor, D. Dowson, Turbulent lubrication theoryapplication to design, J. Lubr. Technol. 96 (1974) 36-47

[6] V.N. Constantinescu, On turbulent lubrication, Proc. Inst. Mech. Eng., London 173 (1959) 881-900

[7] C.W. Ng, C.H.T. Pan, A linearized turbulent lubrication theory, ASME J. Basic Eng. 87 (1965) 675-688

[8] M.K. Ho, J.H. Vohr, Application of energy model of turbulence to calculation of lubricant flows, J. Lubr. Technol. 96 (1974) 95-102

[9] G.G. Hirs, A bulk-flow theory for turbulence in lubricant films, ASME J. Lubr. Technol. 95 (1973) 137-146

[10] J.W. Lund, E.B. Arwas, A simultaneous solution of the lubrication and the energy equations for turbulent journal bearing films. MT1 Rep. No. 64-TR-31, Mech. Tech. Inc. (1964)

[11] V.N. Constantinescu, Basic relations in turbulent lubrication and their extension to include thermal effects, J. Lubr. Technol. 95 (1973) 147-154

[12] Z. Safar, A.Z. Szeri, Thermohydrodynamic lubrication in laminar and turbulent regimes, Trans. ASME, J. Lubr. Technol. 96 (1974) 48-57

[13] Z. Safar, Inertia and thermal effects in turbulent flow journal bearing, Wear 53 (1979) 325-335

[14] H. Hashimoto, S. Wada, Theoretical approach to turbulent lubrication problems including surface roughness effects, Trans, ASME, J. Lubr. Technol. I 11 (1989) 17-22

[15] K. Venkaeswarlu, N.J. Rao, E.V. Venugopal, S. Akella, Three-dimensional laminar and turbulent lubrication in journal bearings, Wear 126 (1990) 263-279

[16] A.K. Tieu, P.B. Kosasih, An expression of Reynolds stress in turbulent lubrication theory. Trans. ASME, J. Lubr. Technol. 114 (1992) 57-60

[17] P.S. Granville A modified Van Driest formula for the mixing length of turbulent boundary layers in pressure gradients, ASME, J. Fluids Eng. Ill (1989) 94-97

[18] D.W. Lee, K.W. Kim, Turbulent lubrication theory using Algebraic Reynolds Stress model in finite journal bearings with cavitation boundary conditions, JSME, Int. J., Series II 33 (1990) 200-207

[19] S.M. Chun, D.H. Ha, Thermohydrodynamic lubrication analysis of high-speed journal bearing considering variable density and variable specific heat, Trib. Int. 37 (2004) 405-413 
[20] Z.C. Peng, M.M. Khonsari, A thermohydrodynamic analysis of foil journal bearings, Trans. ASME, J. Trib. 128 (2006) 534-541

[21] K. Abe, T. Kondoh, Y. Nagano, A new turbulence model for predicting fluid flow and heat transfer in separating and reattaching flows-1. Flow field calculation, Int. J. Heat Mass Transf. 37 (1994) 139-151

[22] K. Mistry, S. Biswas Study of thermal profile and cavitation in a circular journal bearing, Wear 159 (1992) 79-87
[23] H. Heshmat, O. Pinkus, Mixing inlet temperatures in hydrodynamic bearings, ASME J. Trib. 108 (1986) 221-248

[24] M.M. Khonsari, J.Y. Jang, M, Fillon On the generalization of thermohydrodynamic analyses for journal bearings, Trans. ASME, J. Trib. 118 (1996) 571-580

[25] H. Heshmat, The mechanism of cavitation in hydrodynamic lubrication, Trib. Trans. 34 (1991) 177-186

[26] S. Wada, H. Hashimoto, Turbulent lubrication theory using the frictional law, Bull. JSME 22 (1979) 257-263 